\title{
Late Presentation of HIV Infection: Prevalence, Trends, and the Role of HIV Testing Strategies in Guangzhou, China, 2008-2013
}

\author{
Weibin Cheng, ${ }^{1}$ Weiming Tang, ${ }^{2}$ Zhigang Han, \\ Thitikarn May Tangthanasup, ${ }^{2}$ Fei Zhong, ${ }^{1}$ Faju Qin, ${ }^{1}$ and Huifang $\mathrm{Xu}^{1}$ \\ ${ }^{1}$ Department of AIDS/STD Control and Prevention, Guangzhou Center for Disease Control and Prevention, No. 1, \\ Qide Road, Jiahe, Baiyun District, Guangzhou, Guangdong Province 510440, China \\ ${ }^{2}$ University of North Carolina Project-China, No. 2, Lujing Road, Yuexiu District, Guangzhou, Guangdong Province 510095, China \\ Correspondence should be addressed to Huifang Xu; xuhuifang1027@21cn.com
}

Received 7 June 2016; Revised 10 August 2016; Accepted 6 September 2016

Academic Editor: Esteban Martinez

Copyright (C) 2016 Weibin Cheng et al. This is an open access article distributed under the Creative Commons Attribution License, which permits unrestricted use, distribution, and reproduction in any medium, provided the original work is properly cited.

\begin{abstract}
Background. The prevalence, trends, and the role of different HIV testing strategies in late presentation of HIV infection in China were unknown. Methods. Data of newly reported HIV cases in Guangzhou between 2008 and 2013 was analyzed to examine the prevalence, trends, and characteristics of late presentation of HIV infection by three types of HIV testing strategies. Results. Overall, $53.2 \%(1412 / 2653)$ and $27.3 \%$ (724/2653) met the criteria of late presentation and presentation with advanced HIV disease. The overall trend of late presentation of HIV infection within the study period was declining. Late presentation was $62.9 \%$ in 2008 and dropped to $43.3 \%$ in $2013(P<0.001)$; presentation with advanced HIV disease was $40.3 \%$ in 2008 and dropped to $15.2 \%$ in $2013(P<0.001)$. Of the three testing strategies, PITC presented higher odds of both late presentation [AOR (95\% CI): PITC versus VCT: 1.37 (1.09, 1.73); PITC versus MHT: 3.09 (2.16, 4.42)] and presentation with advanced HIV disease [AOR (95\% CI): PITC versus VCT: 1.65 $(1.29,2.11)$; PITC versus MHT: $13.14(8.47,20.39)]$. Conclusions. Although the late presentation of HIV infection was declining, it was still high in Guangzhou. The worse situation among PITC cases urges the policy adjustment in medical settings to increase early HIV diagnosis.
\end{abstract}

\section{Background}

In October 2009, the European Late Presenter Consensus working group reached a consensus definition of late presentation of HIV infection [1]. According to the consensus, late presentation was defined as persons presenting for care with a CD 4 cell count below 350 cells $/ \mu \mathrm{L}$ or presenting with an AIDS-defining event, regardless of the CD4 cell count. Presentation with advanced HIV disease was defined as persons presenting for care with a CD 4 cell count below 200 cells $/ \mu \mathrm{L}$ or presenting with an AIDS-defining event, regardless of the $\mathrm{CD} 4$ cell count. Late presentation for HIV care is detrimental for infected persons and others [2-4]. Studies showed that late presentation not only has a serious impact on HIV transmission [5-8], but also has become the major concern for AIDS and AIDS-related deaths in western countries [9, 10]. Despite tremendous efforts, a substantial number of late
HIV infections still persist worldwide [11-15]. Even though we already have essential data from the United States and European countries, there is a lack of data on late presentation of HIV infection in China and other Asian countries.

A number of studies documented that late presentation of HIV infection is generally more common among marginalized individuals (i.e., ethnic minorities, drug users, and immigrants), people with low perceived risk of HIV infection (e.g., the elderly and heterosexuals), and those of low socioeconomic status (e.g., lower educational attainment) [14, 16-24]. Late presentation of HIV infection reflected the lack of knowledge about HIV and/or cultural and social stigma and socioeconomic barriers which limited the access to healthcare. While HIV testing strategy is considered to be important for late diagnosis as it offers opportunities for HIV positive identification, there is scarce data about testing strategies. 
In the past decade, with the help of international funding agencies, the availability of testing services in China has increased remarkably. In 2004, a free HIV voluntary counseling and testing (VCT) program was introduced to China, which followed the "Four Free, One Care" HIV policy $[25,26]$. VCT services are mainly available at the Centers for Disease Control and Prevention (CDC) and primary healthcare centers that are equipped with trained staff. VCT is free of charge, targeting people who have experienced risk of HIV infection. However, people need to take the initiative to drop by the center to use the service. To increase the VCT service uptake, CDC has implemented a series of intervention programs among the at-risk population, such as men who have sex with men (MSM) and female sex workers. In 2005, mandatory HIV testing (MHT) was launched at detention centers, methadone maintenance treatment clinics, and reeducation/labour camps [27]. MHT was administered to all individuals when they entered into the detention settings. The purpose of MHT was to test at-risk groups including drug users, prisoners, and sex workers along with their clients. In 2006, provider-initiated testing and counseling (PITC) was introduced and implemented at general hospitals and STI clinics in China [26]. HIV test was prescribed by the clinical doctors in the hospital when patients presented with HIV clinical indicator diseases. Meanwhile, PITC included applying HIV testing to individuals who presented to STD clinics. PITC was a paid HIV testing service by using an "opt-out" approach. Patients were informed that HIV testing will take place along with other standard tests and that they must state whether they do not wish to be tested. These three HIV testing strategies are the main types of HIV testing strategies in China. However, according to our knowledge, the effectiveness of testing strategy in late HIV diagnosis has not been evaluated.

Given the clinical and public health benefits of early HIV diagnosis on timely antiretroviral therapy (ART) initiation, it is imperative to understand the situation of late presentation of HIV infection in China and to provide evidence on whichever strategies could facilitate early diagnosis of HIV infection. Thus, by analyzing the HIV case report data in Guangzhou in 2008 to 2013 that was extracted from the Chinese HIV/AIDS Comprehensive Response Information Management System (CRIMS) [28], we conducted this study to examine the prevalence and trend of late presentation of HIV infection and to explore the role of different testing strategies in late presentation of HIV infection.

\section{Methods}

HIV testing service in Guangzhou city is available within authorized CDC, medical institutes, primary healthcare centers, and collective settings. By the end of 2008, there were three HIV confirmatory test laboratories and ninety-one HIV screening laboratories (this increased to 147 in 2013).

Guangzhou started HIV/AIDS case reporting through the Internet in 2005. By the end of 2007, CRIMS was developed and put into use. It is a web-based real-time database system managed by the National Centre for AIDS/STD
Control and Prevention (NCAIDS), China CDC [28]. CRIMS includes case report module and epidemiology follow-up module. An electronic record is created in CRIMS for each patient who tested positive for HIV. Case report and epidemiology follow-up services are conducted by trained staffs from hospitals, district level CDC, and primary healthcare centers.

2.1. Data Sources. The data included in our study was retrieved from CRIMS case report module and epidemiology follow-up module. The data included information on demographic characteristics, sexual and drug use behaviors, transmission routes, medical histories, and laboratory test results. In this study, we only included cases that were over 15 years old when identified and were identified between January 1, 2008, and December 31, 2013, in our data analysis.

2.2. Statistical Analysis. In this study, first-time CD4 cell count results (tested within two weeks after confirmatory HIV positive test) were used to define late presentation of HIV infection. Late presentation was defined as CD4 cell count below 350 cells/ $\mu \mathrm{L}$ or presenting with an AIDSdefining event at the first follow-up, regardless of the CD4 cell count. Presentation with advanced HIV disease was defined as CD 4 cell count below 200 cells/ $\mu \mathrm{L}$ or presenting with an AIDS-defining event at the first follow-up, regardless of the CD4 cell count. Descriptive analyses were used to describe and compare (Chi squared test) demographic characteristics of late presentation of HIV infection cases identified through the three types of testing strategies. Trends of late presentation of HIV infection by three types of testing strategies during the study period were examined. Multivariate logistic regression models were used to compare the proportion of late presentation of HIV infection with different testing strategies. Demographic variables with $P<0.20$ under Chi squared test analysis were adjusted for multivariate logistic regression models. Adjusted odds ratio (AOR) and 95\% confidence intervals (95\% CI) were also estimated. Results with a two-sided $P<0.05$ were considered statistically significant. All statistical analyses were performed with SPSS 18.0 for Windows.

2.3. Ethics Statement. This study was based on data from the Chinese government HIV/AIDS CRIMS. All individuals signed a general consent form when they enrolled in CRIMS, and no additional study informed consent was needed for this current study. The study protocol was approved by Guangzhou CDC Ethics Committee. All personal identification information was removed prior to analysis.

\section{Results}

Of the 6,737 cases identified between 1 January 2008 and 31 December 2013, 2,653 (39.4\%) cases that had CD4 cell count results at the first epidemiology follow-up were included in this study. Demographic characteristics of the cases identified through PITC were tendency to be older (42.1\% aged over 
TABLE 1: Characteristics and proportion of late presentation of HIV infection among newly diagnosed positives by three types of HIV testing strategies between 2008 and 2013 in Guangzhou, China.

\begin{tabular}{|c|c|c|c|c|c|}
\hline \multirow{2}{*}{ Variable } & Total $(N=2653)$ & $\operatorname{VCT}(N=1275)$ & $\operatorname{PITC}(N=832)$ & $\operatorname{MHT}(N=546)$ & \multirow{2}{*}{$P$} \\
\hline & \multicolumn{4}{|c|}{$n(\%)$} & \\
\hline \multicolumn{6}{|l|}{ Year } \\
\hline 2008 & $313(11.8)$ & $156(12.2)$ & $101(12.1)$ & $56(10.3)$ & \multirow{6}{*}{$<0.001$} \\
\hline 2009 & $244(9.2)$ & $79(6.2)$ & $75(9.0)$ & $90(16.5)$ & \\
\hline 2010 & $381(14.4)$ & $95(7.5)$ & $194(23.3)$ & $92(16.8)$ & \\
\hline 2011 & $474(17.8)$ & $206(16.1)$ & $160(19.2)$ & $108(19.8)$ & \\
\hline 2012 & $689(26.0)$ & $382(30.0)$ & $179(21.5)$ & $128(23.4)$ & \\
\hline 2013 & $552(20.8)$ & $357(28.0)$ & $123(14.9)$ & $72(13.2)$ & \\
\hline \multicolumn{6}{|l|}{ Age (years) } \\
\hline$<20$ & $75(2.8)$ & $41(3.2)$ & $28(3.4)$ & $6(1.1)$ & \multirow{5}{*}{$<0.001$} \\
\hline $20 \sim$ & $860(32.4)$ & $535(42.0)$ & $175(21.0)$ & $150(27.5)$ & \\
\hline $30 \sim$ & $981(37.0)$ & $438(34.4)$ & $279(33.5)$ & $264(48.4)$ & \\
\hline $40 \sim$ & $490(18.5)$ & $180(14.1)$ & $207(24.9)$ & $103(18.9)$ & \\
\hline$\geq 50$ & $247(9.3)$ & $81(6.4)$ & $143(17.2)$ & $23(4.2)$ & \\
\hline \multicolumn{6}{|l|}{ Sex } \\
\hline Male & $2264(85.3)$ & $1131(88.7)$ & $612(73.6)$ & $521(95.4)$ & \multirow{2}{*}{$<0.001$} \\
\hline Female & $389(14.7)$ & $144(11.3)$ & $220(26.4)$ & $25(4.6)$ & \\
\hline \multicolumn{6}{|l|}{ Marital status } \\
\hline Single & $1355(51.1)$ & $750(58.8)$ & $228(27.4)$ & $377(69.0)$ & \multirow{4}{*}{$<0.001$} \\
\hline Married & $1015(38.4)$ & $396(31.1)$ & $501(60.2)$ & $118(21.6)$ & \\
\hline Divorced or widowed & $244(9.2)$ & $117(9.2)$ & $91(10.9)$ & $36(6.6)$ & \\
\hline Unknown & $39(1.5)$ & $12(0.9)$ & $12(1.4)$ & $15(2.7)$ & \\
\hline \multicolumn{6}{|l|}{ Educational attainment } \\
\hline Primary school/lower & $526(19.8)$ & $113(8.9)$ & $165(19.8)$ & $248(45.4)$ & \multirow{3}{*}{$<0.001$} \\
\hline Secondary school & $1534(57.8)$ & $677(53.1)$ & $562(67.5)$ & $295(54.0)$ & \\
\hline College/above & $593(22.4)$ & $485(38.0)$ & $105(12.6)$ & $3(0.5)$ & \\
\hline \multicolumn{6}{|l|}{ Route of HIV infection } \\
\hline Male-to-male sexual contact & $917(34.6)$ & $837(65.6)$ & $77(9.3)$ & $3(0.5)$ & \multirow{4}{*}{$<0.001$} \\
\hline Injecting drug use & $654(24.7)$ & $85(6.7)$ & 99 (11.9) & $470(86.1)$ & \\
\hline Heterosexual contact & $967(36.4)$ & $326(25.6)$ & $581(69.8)$ & $60(11.0)$ & \\
\hline Others & $115(4.3)$ & $27(2.1)$ & $75(9.0)$ & $13(2.4)$ & \\
\hline \multicolumn{6}{|l|}{ Place of living } \\
\hline Urban & $1384(52.2)$ & $827(64.9)$ & $316(38.0)$ & $241(44.1)$ & \multirow{3}{*}{$<0.001$} \\
\hline Rural & $480(18.1)$ & $205(17.1)$ & $133(4.4)$ & $133(24.2)$ & \\
\hline Outside of Guangzhou & $789(29.7)$ & $374(45.0)$ & $172(31.5)$ & $172(31.5)$ & \\
\hline \multicolumn{6}{|l|}{ Late presentation } \\
\hline Yes & $1412(53.2)$ & $659(51.7)$ & $590(70.9)$ & $206(37.7)$ & \multirow{2}{*}{$<0.001$} \\
\hline No & $1241(46.8)$ & $616(48.3)$ & $242(29.1)$ & $340(62.3)$ & \\
\hline \multicolumn{6}{|c|}{ Presentation with advanced HIV disease } \\
\hline Yes & $724(27.3)$ & $254(19.9)$ & $416(50.0)$ & $54(9.9)$ & \multirow{2}{*}{$<0.001$} \\
\hline No & $1929(72.7)$ & $1021(80.1)$ & $416(50.0)$ & $492(90.1)$ & \\
\hline
\end{tabular}

VCT: HIV voluntary counseling and testing; PITC: provider-initiated testing and counseling; MHT: mandatory HIV testing.

40), being female (26.4\%), being married (60.2\%), and selfreporting being infected through heterosexual transmission $(69.8 \%)$. VCT cases had higher educational attainment (38.0\% had a college degree or above) and were infected through homosexual contact (65.6\%). The majority of MHT cases were infected through injecting drug use (86.1\%). Overall, $53.2 \%(1412 / 2653)$ and $27.3 \%(724 / 2653)$ were classified into late presentation and presentation with advanced HIV disease groups. PITC cases had a higher proportion of late presentation (70.9\% and $51.7 \%$ for VCT and $37.7 \%$ for MHT) and presentation with advanced HIV disease $(50.0 \%$ and $19.9 \%$ for VCT and $9.9 \%$ for MHT). All characteristics were significantly different among the three groups $(P<0.001)$ (Table 1). 
TABLE 2: Changes in late presentation of HIV infection over time by three types of testing strategies in Guangzhou, 2008-2013.

\begin{tabular}{|c|c|c|c|c|c|c|c|}
\hline & $2008(N=313)$ & $2009(N=244)$ & $2010(N=381)$ & $2011(N=474)$ & $2012(N=689)$ & $2013(N=552)$ & $P$ \\
\hline \multicolumn{8}{|c|}{ Late presentation, $n(\%)$} \\
\hline Total & $197(62.9)$ & $121(49.6)$ & $259(68.0)$ & $260(54.9)$ & $336(48.8)$ & $239(43.3)$ & $<0.001$ \\
\hline \multicolumn{8}{|c|}{ Testing strategy } \\
\hline VCT & $102(65.4)$ & $37(46.8)$ & $55(57.9)$ & $114(55.3)$ & $172(45.0)$ & $136(38.1)$ & $<0.001$ \\
\hline PITC & $78(77.2)$ & $43(57.3)$ & $161(83.0)$ & $106(66.3)$ & $120(67.0)$ & $82(66.7)$ & 0.052 \\
\hline MHT & $17(30.4)$ & $41(45.6)$ & $43(46.7)$ & $40(37.0)$ & $44(34.4)$ & $21(29.2)$ & 0.131 \\
\hline \multicolumn{8}{|c|}{ Presentation with advanced HIV disease, $n(\%)$} \\
\hline Total & $126(40.3)$ & $45(18.4)$ & $166(43.6)$ & $137(28.9)$ & $166(24.1)$ & $84(15.2)$ & $<0.001$ \\
\hline \multicolumn{8}{|c|}{ Testing strategy } \\
\hline VCT & $63(40.4)$ & $11(13.9)$ & $26(27.4)$ & $49(23.8)$ & $63(16.5)$ & $42(11.8)$ & $<0.001$ \\
\hline PITC & $59(58.4)$ & $24(32.0)$ & $126(64.9)$ & $77(48.1)$ & $91(50.8)$ & $39(31.7)$ & 0.002 \\
\hline MHT & $4(7.1)$ & $10(11.1)$ & $14(15.2)$ & $11(10.2)$ & $12(9.4)$ & $3(4.2)$ & 0.294 \\
\hline
\end{tabular}

VCT: HIV voluntary counseling and testing; PITC: provider-initiated testing and counseling; MHT: mandatory HIV testing.

The annual proportions of late presentation from 2008 to 2013 were $62.9 \%$ (197/313), 49.6\% (121/244), 68.0\% (259/680), $54.9 \%$ (260/474), 48.8\% (336/689), and 43.3\% (239/552). Although the overall proportion of late presentation declined during the study period $(P<0.001)$, only the annual rate of late presentation among cases identified through VCT was significantly reduced (from $65.4 \%$ to $38.1 \%$ ). Annual proportions of presentation with advanced HIV disease from 2008 to 2013 were $40.3 \%$ (126/313), $18.4 \%$ (45/244), $28.9 \%$ (1137/680), 24.1\% (166/689), and 15.2\% (84/552). The overall proportion of presentation with advanced HIV disease was declining $(P<0.001)$, while similar results were found for both VCT and PITC strategies (Table 2).

Two multivariate logistic regression models were developed to compare late presentation of HIV infection within three HIV testing strategies. Models were adjusted for year, age, sex, marital status, educational attainment, route of HIV infection, and place of living. Results showed that PITC has the highest odds of late presentation of HIV infection among the three strategies [late presentation, PITC versus VCT, AOR (95\% CI): 1.37 (1.09, 1.73); PITC versus MHT: 3.09 (2.16, 4.42); presentation with advanced HIV disease, PITC versus VCT: 1.65 (1.29, 2.11); PITC versus MHT: 13.14 (8.47, 20.39)]. Among the three strategies, MHT has the lowest odds of late presentation of HIV infection $(P<0.01)$ (Table 3$)$.

\section{Discussion}

In this study, we found that half of the HIV newly diagnosed cases showed late presentation of HIV infection. Among the late presenters, half showed presentation with advanced HIV disease. The epidemic of late presentation of HIV infection in China was high [3], compared to Europe, even though the situation has significantly dropped in the past few years. In addition, this study has compared three types of HIV testing strategies role in the late HIV diagnosis. Results showing a high proportion of late presentation of HIV infection among PITC cases urged the need for improving the capacity of early case detection in medical settings.
Delayed diagnosis of HIV infection may result in late initiation of ART, which subsequently could undermine the effectiveness of clinical outcomes. A recent study demonstrated that deferred initiation of ART at CD4 levels 251 to 350 cells $/ \mu \mathrm{L}$ was associated with higher rates of AIDS and mortality, compared to starting ART at the range of 351 to 450 cells/ $\mu \mathrm{L}$ (HR: $1.28,95 \%$ CI: $1.04-1.57$ ), and that the adverse effect of deferring treatment increased with the decreasing CD4 cell count threshold [29]. Meanwhile, late presentation of $\mathrm{HIV}$ infection reflects the inefficiency of the health providers in response to patients' need for access to HIV services, such as testing and treatment [30]. However, early identification presents an opportunity to encourage safer behavior, which in turn reduces the chance of HIV transmission. The seriousness of late HIV diagnosis in Guangzhou presented a great barrier to achieve the UNAIDS goal of ending AIDS epidemic by 2030.

In this study, robust associations were observed between HIV testing strategies and late presentation of HIV infection. Before that, few reports had explored the association between HIV testing strategies and late HIV diagnosis [18, 31, 32]. Consistent with previous studies, results suggested that PITC did not facilitate more timely diagnosis than targeted HIV counseling. As Duffus WA pointed out, clinical risk-based testing strategy (including PITC), even if implemented successfully in their facility, would still have missed an earlier diagnosis most of the time [33]. PITC acted as an alternative strategy that captured individuals who were unwilling to seek or unaware of seeking HIV testing independently $[31,34]$. However, this "opt-out" testing for HIV following diagnosis of an indicator disease was an important strategy for HIV infection case finding [18]. To increase the efficacy of early diagnosis of HIV infection, researchers suggested that adjusting routine provider-initiated HIV testing for atrisk groups in settings such as sexually transmitted infection clinics, drug dependency programs, or antenatal care might be more effective [35].

Interestingly, mandatory HIV testing showed great potential in identifying cases early. In Guangzhou, mandatory testing was well targeted at drug users. The ongoing 
TABLE 3: Comparison of different testing strategies on late presentation of HIV infection by multivariate logistic regression ${ }^{+}$.

\begin{tabular}{lcccc}
\hline & $\begin{array}{c}\text { Late presentation } \\
\text { AOR }(95 \% \mathrm{CI})\end{array}$ & $P$ & $\begin{array}{c}\text { Presentation with advanced HIV disease } \\
\text { AOR }(95 \% \mathrm{CI})\end{array}$ & $P$ \\
\hline VCT & Ref. & & Ref. & \\
PITC & $1.37(1.09,1.73)$ & 0.008 & $1.65(1.29,2.11)$ & $<0.001$ \\
MHT & $0.32(0.23,0.46)$ & $<0.001$ & $0.13(0.08,0.20)$ & $<0.001$ \\
\hline PITC versus MHT & $3.09(2.16,4.42)$ & $<0.001$ & $13.14(8.47,20.39)$ & $<0.001$ \\
\hline
\end{tabular}

AOR: adjusted odds ratio; CI: confidence intervals.

VCT: HIV voluntary counseling and testing; PITC: provider-initiated testing and counseling; MHT: mandatory HIV testing.

${ }^{+}$Models were adjusted for year, age, sex, marital status, educational attainment, route of HIV infection, and place of living.

mandatory HIV testing among drug users increased early diagnosis of HIV infection among this population and significantly contributed to the control of HIV epidemic among intravenous drug users in Guangzhou, as well as across China [36]. This result also concurred with the finding of one study conducted in the Republic of Korea [32]. Furthermore, studies indicated that mandatory HIV testing allows healthcare providers to provide early intervention to the at-risk population and to provide opportunities for testing, substance abuse treatment, and ART, which played an important role in the control of HIV epidemic [37, 38].

China Nation Free Antiretroviral Therapy Program (NFATP) was piloted in 2002 and was implemented nationally in 2003. Free ART criteria were started at CD4 cell count of less than 200 cells $/ \mu \mathrm{L}$ and increased to less than 350 cells $/ \mu \mathrm{L}$ in 2008 [39]. Although the longitudinal trends of late presentation of HIV infection in this study had shown a declining gesture, persistently high prevalence of late presentation (about 60\%) was observed among PITC. One important reason is that high level of HIV related stigma/discrimination and low HIV risk perception among at-risk populations hindered the initiative access to HIV services [40]. PITC was mainly applied for the general population in the medical settings, which presented a gap of demanding for intervention to raise HIV awareness among at-risk individuals in the general population. Another common reason for those presenting late was that patients have often been seen with indicator diseases in the recent past by healthcare professionals without prompt offering of an HIV test [41]. These represent missed opportunities for diagnosis. HIV testing should continue to expand across clinical settings to reduce the number of patients living with undiagnosed HIV infection [42].

The limitations of the current reported study include the following: first, more than half of the cases were excluded due to the lack of CD4 cell count results which may compose an unrepresentative sample. Nonetheless, we compared cases that had CD4 cell count result at first follow-up with those that did not; a minor difference of the proportion of cases that progressed to AIDS at the end of the observation year was found between these two groups $(32.2 \%$ versus 29.1\%). Second, the definition of late presentation of HIV infection used in this study was relatively conservative. The interval between HIV diagnosis and CD4 cell count test within two weeks could underestimate and make comparison difficult. However, evidence suggested that although the percentage of HIV-infected persons with late diagnosis increases with longer cut-offs, the general factors associated with late diagnosis may not vary substantially across the different cut-offs [43]. Third, stigma and social desirability may prevent drug users, sex workers, and homosexual men from reporting the transmission route correctly, and this may lead to information bias. Nonetheless, these three HIV testing strategies have been designed to target different population at risk of HIV infection. Understanding the efficiency of different testing strategies on early case finding would be useful for HIV prevention policy adjustment and resources allocation.

Even with these limitations, this study presented important data that pointed out a high prevalence of late presentation of HIV infection among HIV cases identified in Guangzhou. Furthermore, our data demonstrated that voluntary HIV counseling and testing strategies were more effective in early HIV diagnosis. Recently, the national antiretroviral therapy guideline updated initial ART to treat all patients once diagnosis of HIV infection is confirmed. The early identification of HIV infection cases enables treatment to be initiated at a time when responses are optimal, which in turn reduces the chance of transmission [39]. However, more efforts to better understand the optimal HIV testing practice to facilitate early HIV diagnosis are needed.

\section{Abbreviations}

HIV: Human immunodeficiency virus

AIDS: Acquired immune deficiency syndrome

VCT: HIV voluntary counseling and testing

PITC: $\quad$ Provider-initiated testing and counseling

MHC: $\quad$ Mandatory HIV testing

STI: $\quad$ Sex transmitted infection

CDC: $\quad$ Centers for Disease Control and Prevention

ART: Antiretroviral therapy

CRIMS: Chinese HIV/AIDS Comprehensive

Response Information Management System

NCAIDS: National Centre for AIDS/STD Control and Prevention

STD: $\quad$ Sex transmitted disease

AOR: $\quad$ Adjusted odds ratio

CI: Confidence intervals

HR: Hazard ratio

NFATP: Nation Free Antiretroviral Therapy Program. 


\section{Disclosure}

Funding organizations had no role in the design and conduct of the study; collection, management, analysis, and interpretation of the data; and preparation, review, or approval of the manuscript.

\section{Competing Interests}

The authors have no competing interests to report.

\section{Authors' Contributions}

Weibin Cheng developed study protocol, analyzed data, interpreted findings, and drafted and revised the manuscript. Weiming Tang and Thitikarn May Tangthanasup revised the manuscript and checked the language. Zhigang Han prepared the study proposal, supervised data collection, and interpreted the findings. Fei Zhong analyzed data and interpreted the findings. Faju Qin collected data and performed data management. Huifang Xu supervised the study and provided technical inputs to the manuscript. All authors critically reviewed and approved the final version of this paper for publication.

\section{Acknowledgments}

The authors are indebted to the staff from the Twelve Districts Centers for Disease Control and Prevention for their contribution to the case report and follow-up. The funding of this study was supported by the Project for Key Medicine Discipline Construction of Guangzhou Municipality (Grant no. 2017-2019-04), Medical Scientific Research Foundation of Guangdong Province (A2013539), Medical Scientific Program of Guangzhou (20141A011061), and Science and Technology Program of Guangzhou (2012Y2-00021).

\section{References}

[1] A. Antinori, T. Coenen, D. Costagiola et al., "Late presentation of HIV infection: a consensus definition," HIV Medicine, vol. 12, no. 1, pp. 61-64, 2011.

[2] M. Limb, "More routine HIV testing is needed to reduce late diagnoses, says public health agency," British Medical Journal, vol. 343, Article ID d7794, 2011.

[3] A. Mocroft, J. D. Lundgren, M. L. Sabin et al., "Risk factors and outcomes for late presentation for HIV-positive persons in Europe: results from the Collaboration of Observational HIV Epidemiological Research Europe Study (COHERE)," PLoS Medicine, vol. 10, no. 9, Article ID e1001510, 2013.

[4] R. O. Valdiserri, "Late HIV diagnosis: bad medicine and worse public health," PLoS Medicine, vol. 4, article e200, 2007.

[5] G. Marks, N. Crepaz, and R. S. Janssen, "Estimating sexual transmission of HIV from persons aware and unaware that they are infected with the virus in the USA," AIDS, vol. 20, no. 10, pp. 1447-1450, 2006.

[6] M. Fisher, "Late diagnosis of HIV infection: major consequences and missed opportunities," Current Opinion in Infectious Diseases, vol. 21, no. 1, pp. 1-3, 2008.
[7] G. D. Sanders, A. M. Bayoumi, V. Sundaram et al., "Costeffectiveness of screening for HIV in the era of highly active antiretroviral therapy," The New England Journal of Medicine, vol. 352 , no. 6, pp. 570-585, 2005.

[8] H. I. Hall, D. R. Holtgrave, and C. Maulsby, "HIV transmission rates from persons living with HIV who are aware and unaware of their infection," AIDS, vol. 26, no. 7, pp. 893-896, 2012.

[9] J. Brännström, B. Åkerlund, M. Arneborn, A. Blaxhult, and J. Giesecke, "Patients unaware of their HIV infection until AIDS diagnosis in Sweden 1996-2002-a remaining problem in the highly active antiretroviral therapy era," International Journal of STD and AIDS, vol. 16, no. 10, pp. 702-706, 2005.

[10] B. Castelnuovo, E. Chiesa, S. Rusconi et al., "Declining incidence of AIDS and increasing prevalence of AIDS presenters among AIDS patients in Italy," European Journal of Clinical Microbiology and Infectious Diseases, vol. 22, no. 11, pp. 663-669, 2003.

[11] A. K. Sullivan, H. Curtis, C. A. Sabin, and M. A. Johnson, "Newly diagnosed HIV infections: review in UK and Ireland," British Medical Journal, vol. 330, no. 7503, pp. 1301-1302, 2005.

[12] "Late versus early testing of HIV-16 sites, United States, 20002003," Morbidity and Mortality Weekly Report, vol. 52, no. 25, pp. 581-586, 2003.

[13] T. Shivaji, A. Diniz, and H. Cortes-Martins, "Characteristics of late presentation of HIV infection in MSM and heterosexual adults in Portugal 2011-2013," Journal of the International AIDS Society, vol. 17, no. 4, supplement 3, Article ID 19690, 2014.

[14] M. J. Trepka, K. P. Fennie, D. M. Sheehan, K. Lutfi, L. Maddox, and S. Lieb, "Late HIV diagnosis: differences by rural/urban residence, Florida, 2007-2011," AIDS Patient Care and STDs, vol. 28, no. 4, pp. 188-197, 2014.

[15] K. D. Wilson, R. Dray-Spira, C. Aubrière, C. Hamelin, B. Spire, and F. Lert, "Frequency and correlates of late presentation for HIV infection in France: older adults are a risk group-results from the ANRS-VESPA2 study, France," AIDS Care, vol. 26, supplement 1, pp. S83-S93, 2014.

[16] C. M. Carrizosa, E. J. Blumberg, M. F. Hovell et al., "Determinants and prevalence of late HIV testing in Tijuana, Mexico," AIDS Patient Care and STDs, vol. 24, no. 5, pp. 333-340, 2010.

[17] H. I. Hall, J. Halverson, D. P. Wilson et al., "Late diagnosis and entry to care after diagnosis of human immunodeficiency virus infection: a country comparison," PLoS ONE, vol. 8, no. 11, Article ID e77763, 2013.

[18] P. Scognamiglio, G. Chiaradia, G. De Carli et al., "The potential impact of routine testing of individuals with HIV indicator diseases in order to prevent late HIV diagnosis," BMC Infectious Diseases, vol. 13, article 473, 2013.

[19] D. Klein, L. B. Hurley, D. Merrill, and C. P. Quesenberry Jr., "Review of medical encounters in the 5 years before a diagnosis of HIV-1 infection: implications for early detection," Journal of Acquired Immune Deficiency Syndromes, vol. 32, no. 2, pp. 143152, 2003.

[20] M. J. Mugavero, C. Castellano, D. Edelman, and C. Hicks, "Late diagnosis of HIV infection: the role of age and sex," The American Journal of Medicine, vol. 120, no. 4, pp. 370-373, 2007.

[21] C. Delpierre, R. Dray-Spira, L. Cuzin et al., "Correlates of late HIV diagnosis: implications for testing policy," International Journal of STD and AIDS, vol. 18, no. 5, pp. 312-317, 2007.

[22] K. Mojumdar, M. Vajpayee, N. K. Chauhan, and S. Mendiratta, "Late presenters to HIV care and treatment, identification of associated risk factors in HIV-1 infected Indian population," BMC Public Health, vol. 10, article 416, 2010. 
[23] I. M. Kigozi, L. M. Dobkin, J. N. Martin et al., "Late-disease stage at presentation to an HIV clinic in the era of free antiretroviral therapy in Sub-Saharan Africa," Journal of Acquired Immune Deficiency Syndromes, vol. 52, no. 2, pp. 280-289, 2009.

[24] P. K. Drain, E. Losina, G. Parker et al., "Risk factors for late-stage HIV disease presentation at initial HIV diagnosis in Durban, South Africa," PLoS ONE, vol. 8, no. 1, Article ID e55305, 2013.

[25] B. Gazzard, N. Clumeck, A. d'Arminio Monforte, and J. D. Lundgren, "Indicator disease-guided testing for HIV-the next step for Europe?” HIV Medicine, vol. 9, supplement 2, pp. 34-40, 2008.

[26] WHO and UNAIDS, Guidance on Provider-Initiated HIV Testing and Counselling in Health Facilities, World Health Organization, Geneva, Switzerland, 2007.

[27] Z. Wu, X. Sun, S. G. Sullivan, and R. Detels, "Public health. HIV testing in China," Science, vol. 312, no. 5779, pp. 1475-1476, 2006.

[28] Y. Mao, Z. Wu, K. Poundstone et al., "Development of a unified web-based national HIV/AIDS information system in China," International Journal of Epidemiology, vol. 39, supplement 2, Article ID dyq213, pp. ii79-ii89, 2010.

[29] J. A. Sterne, M. May, D. Costagliola et al., "Timing of initiation of antiretroviral therapy in AIDS-free HIV-1-infected patients: a collaborative analysis of 18 HIV cohort studies," The Lancet, vol. 373, pp. 1352-1363, 2009.

[30] T. Coenen, J. Lundgren, J. V. Lazarus, and S. Matic, "Optimal HIV testing and earlier care: the way forward in Europe," HIV Medicine, vol. 9, supplement 2, pp. 1-5, 2008.

[31] S. M. Topp, M. S. Li, J. M. Chipukuma et al., "Does providerinitiated counselling and testing (PITC) strengthen early diagnosis and treatment initiation? Results from an analysis of an urban cohort of HIV-positive patients in Lusaka, Zambia," Journal of the International AIDS Society, vol. 15, no. 2, Article ID 17352, 2012.

[32] P. G. Choe, W. B. Park, J. S. Song et al., "Late presentation of HIV disease and its associated factors among newly diagnosed patients before and after abolition of a government policy of mass mandatory screening," Journal of Infection, vol. 63, no. 1, pp. 60-65, 2011.

[33] W. A. Duffus, K. Weis, L. Kettinger, T. Stephens, H. Albrecht, and J. J. Gibson, "Risk-based HIV testing in South Carolina health care settings failed to identify the majority of infected individuals," AIDS Patient Care and STDs, vol. 23, no. 5, pp. 339345, 2009.

[34] D. R. Holtgrave, "Costs and consequences of the US centers for disease control and prevention's recommendations for opt-out HIV testing," PLoS Medicine, vol. 4, no. 6, pp. 1011-1018, 2007.

[35] Y. Yazdanpanah, J. Lange, J. Gerstoft, and G. Cairns, "Earlier testing for HIV—how do we prevent late presentation?" Antiviral Therapy, vol. 15, supplement 1, pp. 17-24, 2010.

[36] L. Wang, W. Guo, D. Li et al., "HIV epidemic among drug users in China: 1995-2011," Addiction, vol. 110, no. 1, pp. 20-28, 2015.

[37] S. Basu, D. Smith-Rohrberg, S. Hanck, and F. L. Altice, "HIV testing in correctional institutions: evaluating existing strategies, setting new standards," AIDS and Public Policy Journal, vol. 20, no. 1-2, pp. 3-24, 2005.

[38] A. A. Amankwaa, L. C. Amankwaa, and C. O. Ochie Sr., "Revisiting the debate of voluntary versus mandatory HIV/AIDS testing in U.S. prisons," Journal of Health and Human Services Administration, vol. 22, no. 2, pp. 220-236, 1999.

[39] F. Zhang, Z. Dou, Y. Ma et al., "Effect of earlier initiation of antiretroviral treatment and increased treatment coverage on
HIV-related mortality in China: a national observational cohort study," The Lancet Infectious Diseases, vol. 11, no. 7, pp. 516-524, 2011.

[40] S. Arreola, G.-M. Santos, J. Beck et al., "Sexual stigma, criminalization, investment, and access to HIV services among men who have sex with men worldwide," AIDS and Behavior, vol. 19, no. 2, pp. 227-234, 2015.

[41] S. Ellis, H. Curtis, and E. L. C. Ong, "HIV diagnoses and missed opportunities. Results of the British HIV Association (BHIVA) National Audit 2010," Clinical Medicine, vol. 12, no. 5, pp. 430434, 2012.

[42] A. Palfreeman, M. Fisher, E. Ong et al., "Testing for HIV: concise guidance," Clinical Medicine, vol. 9, no. 5, pp. 471-476, 2009.

[43] B. Yang, S. K. Chan, N. Mohammad et al., "Late HIV diagnosis in Houston/Harris County, Texas, 2000-2007," AIDS Care, vol. 22, no. 6, pp. 766-774, 2010. 


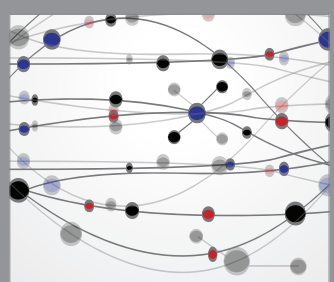

The Scientific World Journal
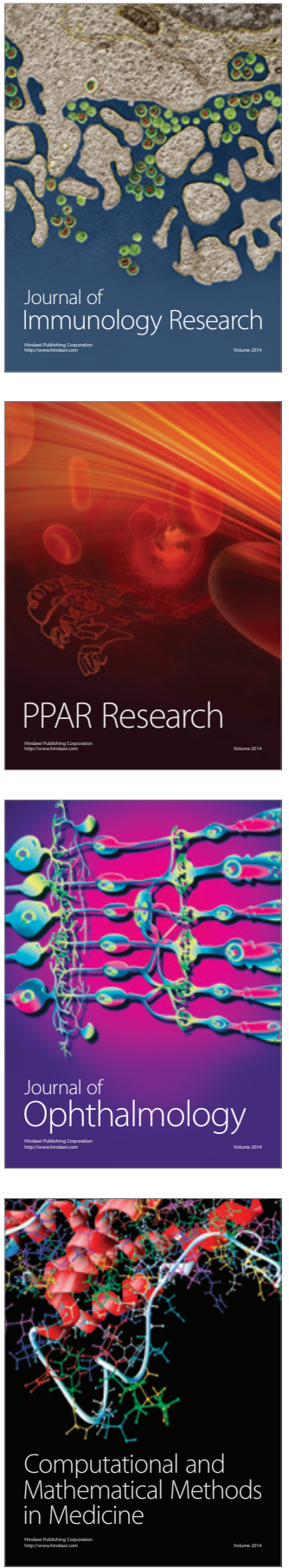

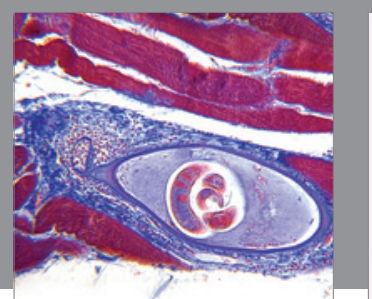

Gastroenterology Research and Practice

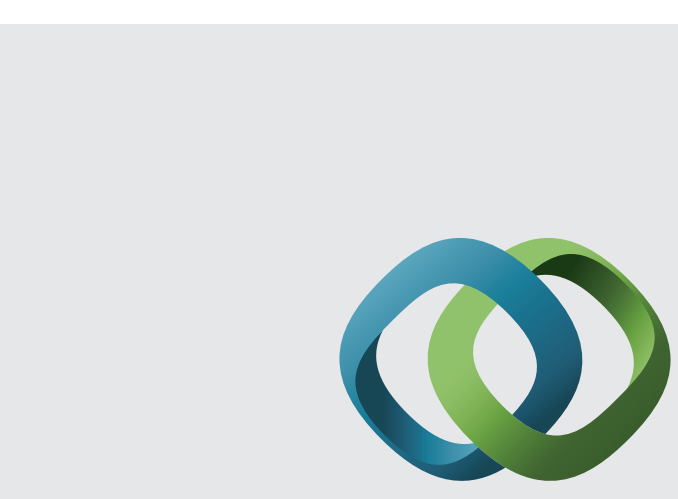

\section{Hindawi}

Submit your manuscripts at

http://www.hindawi.com
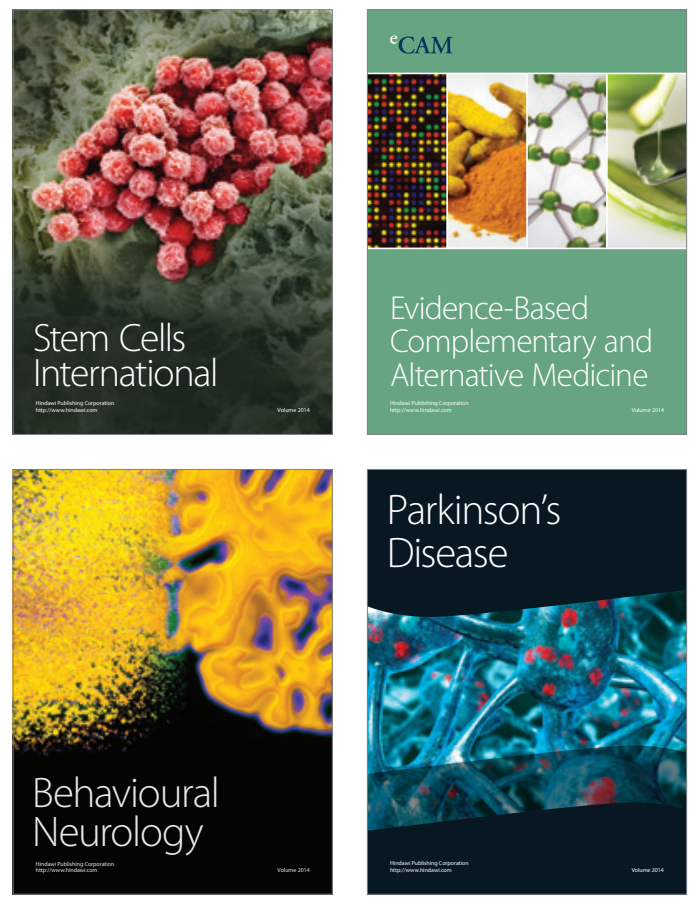
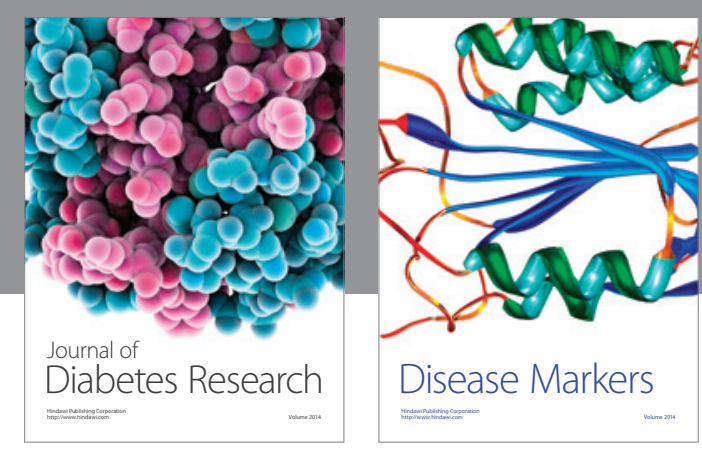

Disease Markers
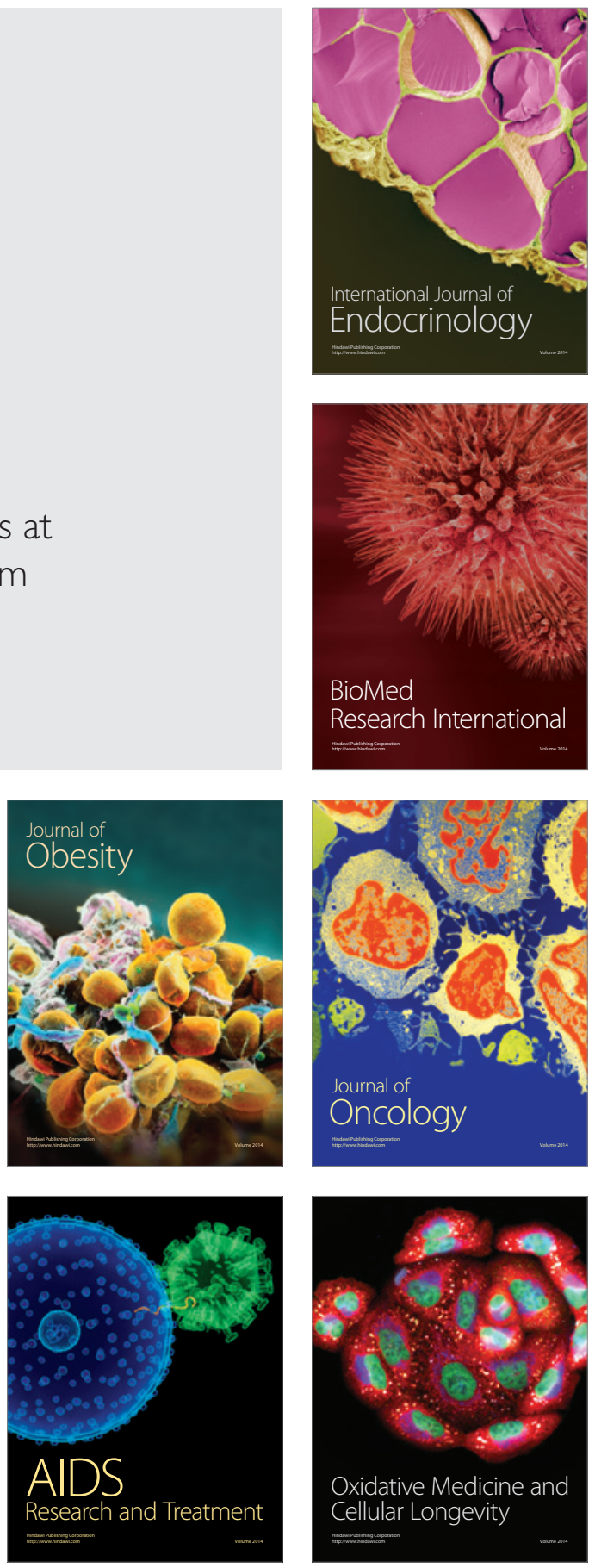